\title{
CAPILLARY OBSERVATIONS IN PATIENTS WITH HEMORRHAGIC FEVER AND OTHER INFECTIOUS ILLNESSES ${ }^{1}$
}

\author{
By SHELDON E. GREISMAN \\ (From the Departments of Medicine and Microbiology, University of Maryland School of \\ Medicine, Baltimore, Md., and the Hemorrhagic Fever Center, Korea)
}

(Submitted for publication May 21, 1957; accepted August 20, 1957)

Although the capillary vascular system is known to be affected during many infectious illnesses (1-5), the in vivo study of the small blood vessels in man during infection has been almost entirely neglected. Certain infectious diseases are especially characterized by clinical syndromes which can be largely or entirely attributable to a generalized dysfunction of the vascular system (6-8); a study of the reaction of the small blood vessels in these states might therefore be particularly significant. One such illness, hemorrhagic fever, was selected initially for the in vivo study of the capillary alterations produced by infectious agents in humans. Hemorrhagic fever was first encountered by Western physicians during the Korean Campaign. A classical case usually proceeded through four rather distinct phases; namely, 1) an initial acutely febrile onset, persisting for an average of three to five days, 2) a hypotensive or shock phase often developing during defervescence and persisting from a few hours to several days, 3) a hypertensive oliguric stage lasting three to five days on the average, and 4) a diuretic phase merging into convalescence (9). The deeply flushed appearance, the petechiae and ecchymosis of the skin, conjunctiva and hard palate, and the strongly positive Rumpel-Leede test, all occurring early in the disease $(10,11)$, suggested the occurrence of a profound and generalized alteration in the function of the small blood vessels. The characteristic postmortem findings, consisting chiefly of intense generalized capillary dilatation, focal hemorrhages, and edema in the loose areolar tissues, strongly support this concept (12-14). Indeed many, if not most, of the clinical features which develop after the febrile phase can be accounted for as the consequences of generalized capillary dysfunction.

\footnotetext{
1 This study was sponsored by the Commission on Hemorrhagic Fever and supported in part by the Armed Forces Epidemiological Board by a contract with the Office of the Surgeon General.
}

This report describes the changes detected during serial observations of the nail-fold capillary bed of such patients with hemorrhagic fever. The capillary alterations observed in patients with other infectious diseases who were studied in a similar manner are also described. The findings indicate that significant alterations of the capillary vessels may be observed directly during hemorrhagic fever and during certain other infectious illnesses, and that these capillary alterations can be correlated with the clinical course and pathological findings.

\section{MATERIALS AND METHODS}

\section{Selection of patients}

Random and serial capillary studies were performed on 190 consecutive United Nations soldiers admitted to the Forty-eighth Mobile Army Surgical Hospital in Korea during the fall of 1953 because of febrile disease. The subjects, ranging in age from 18 to 45 , were usually transferred from Division Clearing Station during the third or fourth day of illness with the tentative diagnosis of "hemorrhagic fever, suspect." Eighty of this group were rejected because the observer was unable to visualize the nail-fold capillary vessels adequately (negroid pigmentation, thickened cuticles) or because of local pathologic alterations (clubbing, chronic inflammation, traumatization). The diagnosis of hemorrhagic fever was eventually confirmed in 71 patients; 39 patients were found to have various other acute febrile illnesses. Nine of a group of young male adult patients in the United States with $Q$ fever studied by Tigertt and Benenson (15) and three patients with Rocky Mountain spotted fever were subsequently included in the present study (Table I). ${ }^{2}$

\section{Selection of capillary bed}

Two areas are readily accessible for in vivo capillary examination in the human subject-the bulbar conjunctiva and the skin of the nail-fold. Since the vessels of the bulbar conjunctiva are often grossly injected during

2 The capillary studies pertaining to $Q$ fever were performed in collaboration with Dr. Charles L. Wisseman, Professor of Microbiology, University of Maryland School of Medicine. 
many febrile or upper respiratory illnesses, and since preliminary observations indicated minimal nail-fold capillary alterations as a result of fever or respiratory illness per se, the nail-fold bed was selected for further capillary studies. Moreover, the utilization of the nailfold capillary bed ensured that the portion of the minute vascular system selected for study was anatomically and functionally comparable in all patients.

\section{Method of examination}

The patients were examined in the recumbent position, with the extended arm at the level of the sternum. Cedar oil was applied to the base of the nail-fold of the fourth finger of the left hand and the terminal capillary loops visualized with a Leitz capillary microscope at a magnification of $80 \times$. As it was impossible to maintain constant room temperatures, two heavy blankets were placed over the patients to induce reflex vasodilatation. No observations were made until the fingers remained constantly warm to the touch.

\section{Criteria of capillary changes}

Four indices of nail-fold capillary alterations were followed daily:

1) Degree of capillary dilatation. The capillary vessels of the nail-fold are arranged in parallel hairpin shaped loops which vary considerably in length and width. The majority of these loops are not uniform in caliber, but gradually increase in diameter during the transition from arterial to venous segments. Although the walls of the capillary loops are invisible, the absence of any significant marginal plasma zone permits the true diameter to be accurately gauged from the width of the capillary blood stream (16). After study of several microscopic fields comprising a total of approximately 50 to 75 vessels, 8 to 10 representative loops were selected and the widest portions of these vessels (invariably the venous segments) measured with an ocular micrometer. With increased experience, this technique was found to provide a reproducible index of mean widest capillary diameter which generally fell within \pm 3 micra of the value obtained by averaging the sum of the widest segments of the individual capillary diameters. Although less sensitive to caliber alterations, such a technique was distinctly more practical than the cinematographic analysis utilized by Crawford (16), wherein individual nail-fold capillary loops were found to vary in diameter from moment to moment, the venous segment changes normally not exceeding \pm 1.5 micra.

The widest (venous) segment diameters were utilized for this study since previous observations in approximately three hundred persons with various noninfectious illnesses indicated that such measurements generally reflected the largest increases in capillary diameter during reaction to injury (anoxia, cold, trauma) (17). Comparable observations have previously been reported for Raynaud's disease (18), polycythemia vera (19), and congestive heart failure (20).
TABLE I

Diagnosis in 51 febrile patients with diseases other than hemorrhagic fever

\begin{tabular}{cl}
\hline $\begin{array}{c}\text { No. of } \\
\text { patients }\end{array}$ & \multicolumn{1}{c}{ Diagnosis } \\
\hline 14 & Fever of unknown origin \\
9 & Q fever \\
6 & Acute tonsillitis \\
5 & Acute sinusitis \\
4 & Pneumococcal lobar pneumonia \\
4 & Infectious hepatitis \\
3 & Acute vivax malaria \\
1 & Infectious mononucleosis \\
1 & Generalized urticaria \\
1 & Scrub typhus \\
3 & Rocky Mountain spotted fever \\
\hline
\end{tabular}

2) Capillary vasomotor activity. Intermittent gaps in blood flow normally occur in the nail-fold capillary loops. These gaps in blood flow probably reflect intermittent vasoconstriction of the arterial segments of the capillary loops or of the parent arterioles (21), thus constituting an index of vasomotor activity of the minute vascular bed. Although the gaps in blood flow in any given vessel were irregular and occurred independently of those seen in adjacent capillary vessels, the average number of interruptions in blood flow per minute, as determined in five adjacent vessels, provided a reasonably reproducible index of capillary vasomotor activity. The results of such a study, however, cannot be considered as an absolute indication of capillary vasomotor activity, since the "gaps" in capillary blood flow may not be due to interruptions in blood flow but may also represent changes in arteriolar axial streaming of red blood cells with capillary plasma "skimming."

3) Rate of capillary blood flow. With experience it was found that estimates of stagnation, slowed, normal, or accelerated capillary blood flow were consistent in a given patient at a given time and that comparisons between patients or in one patient at different times were valid. Such estimates provided information which was as useful to the present study as were accurately timed measurements.

4) Sludging. Normally, red blood cells flow homogeneously through the capillary bed. In some disease states they adhere to each other in the form of irregular globules constituting microscopic emboli known as sludge (22). The intensity of sludging was observed and graded from 0 to 4 plus.

\section{Response to l-norepinephrine}

A continuous intravenous infusion of $l$-norepinephrine was given to hemorrhagic fever patients during the phase of hypotension (diastolic arterial pressures under $50 \mathrm{~mm}$. $\mathrm{Hg}$ ) and to 8 nonhypotensive hemorrhagic fever patients during the first afebrile day. The infusion of $l$-norepinephrine was increased at 10 minute intervals by increments of 0.05 to 0.07 gamma per $\mathrm{Kg}$. per minute. With each increment the response of the nail-fold capillary bed and of the arterial blood pressure was recorded. 


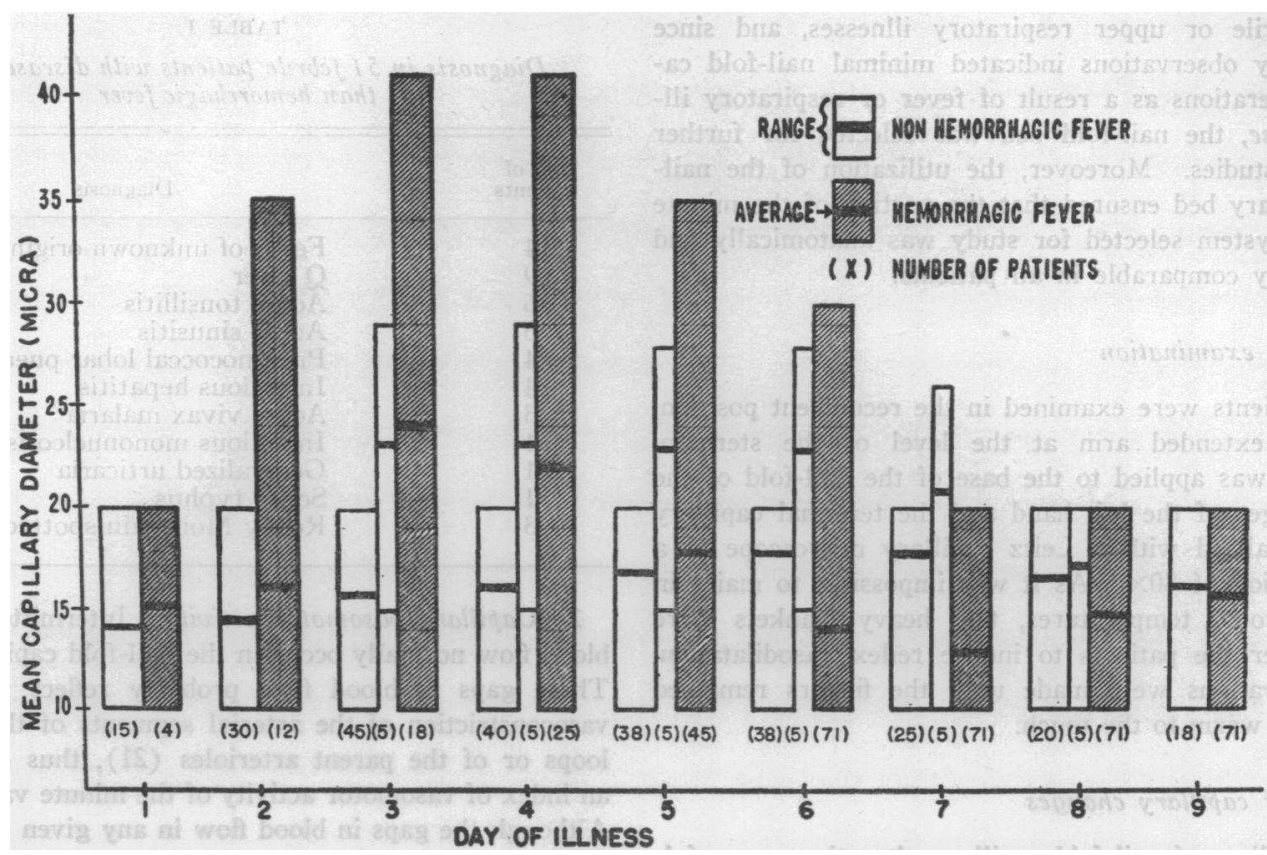

Fig. 1. Serial Observations of Mean Widest Nall-Fold Capillary Diameter in Patients With Hemorrhagic Fever and With the Other Infectious Illnesses Listed in TABLE I

Center column indicates mean widest capillary diameter changes in the patients with Rocky Mountain spotted fever, scrub typhus, and infectious mononucleosis. (The apparent fixation of the lower range of capillary diameter at 10 micra represents those patients with initial values at 10 micra who manifested no nail-fold capillary alterations throughout illness.)

\section{Evaluation of capillary changes}

Control baseline nail-fold capillary observations in hemorrhagic fever patients were unobtainable as all patients were clinically ill at the time of admission. Indeed, only twelve patients were examined prior to the third day of illness. Evaluation of the capillary findings during the febrile and hypotensive phases of hemorrhagic fever was therefore based upon 1) serial alterations of the capillary bed as the illness progressed and subsequently during convalescence, and 2) comparison with groups of patients with infectious diseases other than hemorrhagic fever. The previous nail-fold capillary examinations performed in approximately three hundred persons with various noninfectious illnesses (17) served as the background experience for these capillary studies and permitted on over-all evaluation of the vascular changes observed during the present investigation.

\section{RESULTS}

\section{Hemorrhagic fever}

A) Changes observed in the nail-fold capillary bed during the course of hemorrhagic fever

1) Capillary dilatation. The mean widest capillary diameter on the first day of illness of patients with hemorrhagic fever and those with other febrile diseases fell within a range of 10 to 20 micra, with an over-all average of 15 micra. This is comparable to observations by Crawford that the majority of capillary venous segments in the normal nail-fold bed measure 15 to 17 micra in diameter (16). Progressive capillary dilatation involving both the arterial and venular segments of the capillary loops developed in the patients with hemorrhagic fever. Dilatation of the venular segments was always the more pronounced. Increases in mean widest capillary diameter were usually maximum during the third or fourth days of illness (Figure 1). At this stage, seven of the patients (10 per cent) with hemorrhagic fever developed minute hemorrhages in the capillary bed consisting of red blood cells surrounding the length of the vessel or consolidated hemorrhages, usually at the tip of the capillary loop. In the five subjects with hemorrhages surrounding the length of the capillary, the red blood cells were distributed in single or double file and it was postulated that the cells had escaped by a process of diapedesis; in 
the two patients with consolidated hemorrhages, an actual rupture of the capillary loops was indicated by cessation of blood flow distal to the hemorrhage with diversion of the blood flow into the hemorrhage site.

By the fifth day of hemorrhagic fever, generalized dilatation of the nail-fold capillary bed had usually begun to subside, though isolated dilatation of one or more venular segments frequently persisted for one to two weeks. These dilatations were particularly striking in their abrupt demarcation from the arterial segments, thereby resembling microaneurisms. In those patients who developed hypotension, dilatation of the nail-fold capillary bed was often marked and extended beyond the fourth day of illness (Figure 2). The capillary dilatation usually persisted throughout the duration of the hypotensive period.

By the sixth day of hemorrhagic fever, capillary constriction appeared, and during the seventh and eighth days of illness, the capillary vessels attained their narrowest caliber. Although this constriction involved the arterial segments of the capillary loops most severely, the venular segments were also attenuated. In patients who developed significant hypertension, the capillary bed became severely constricted, though intense constriction also occurred in the absence of clinical hypertension.

By the ninth or tenth day of illness, the diam-

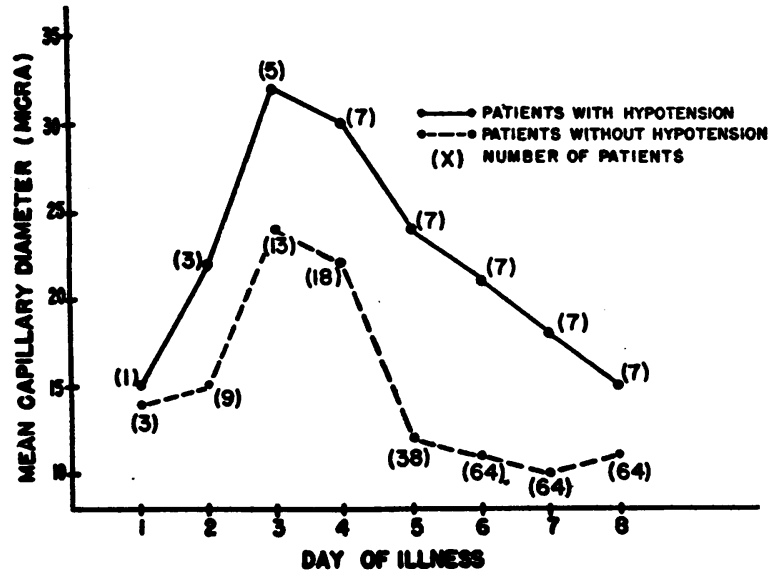

Fig. 2. Serral Changes in Mran Widest Nall-Fold Capiluary Duameter in Hemorrhagic Fever Patients With and Without Clinical Evidence of HypoTENSION

eters of the arterial and venous capillary segments generally began to return towards normal. Serial photomicrographic tracings of nail-fold capillary diameter changes in a representative patient with a moderately severe case of hemorrhagic fever are shown in Figure 3.

2) Capillary vasomotor activity. The intensity of capillary vasomotor activity (and/or plasma "skimming") during hemorrhagic fever exhibited an inverse relation to the severity of capillary dilatation. During the initial four days of illness, few

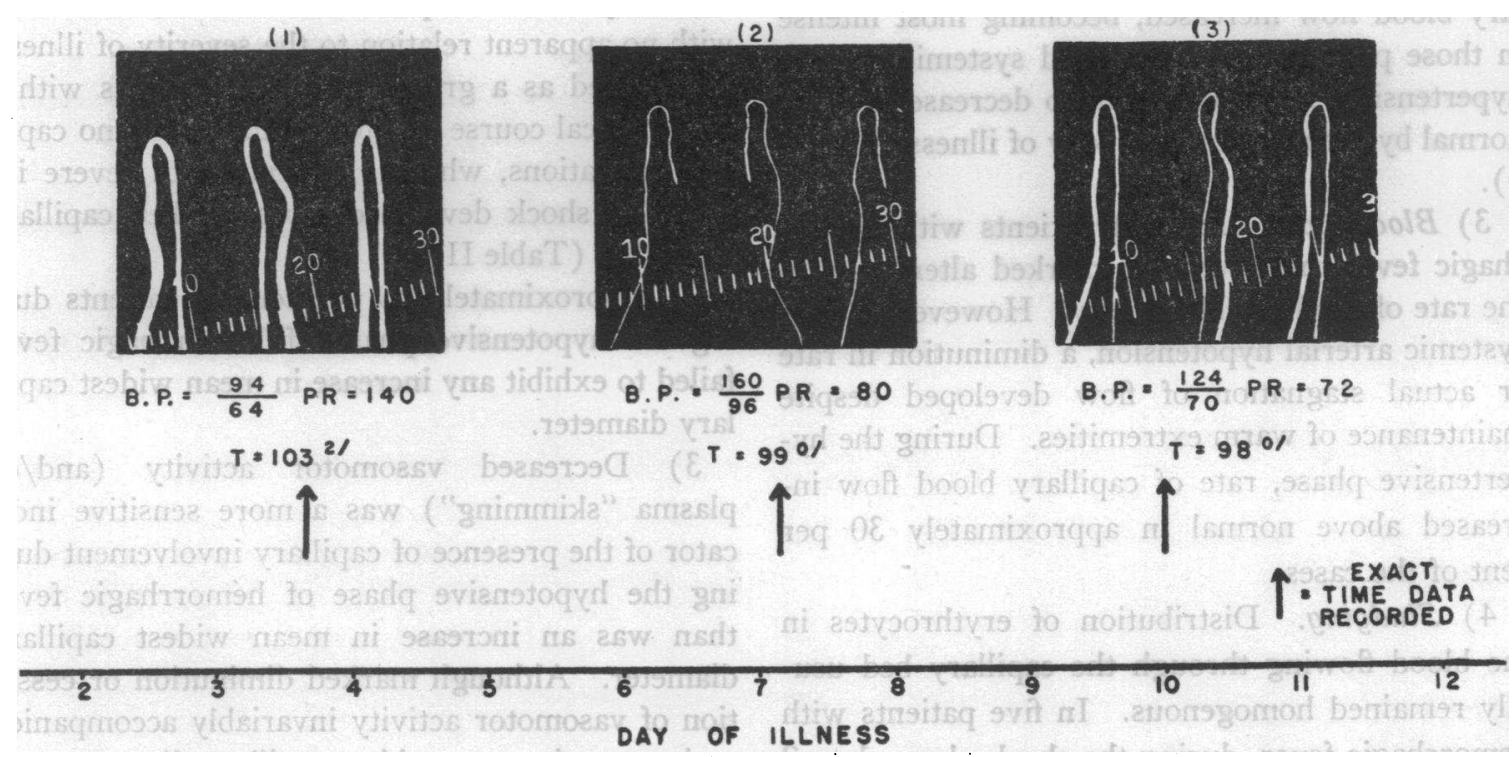

Fig. 3. Serial Photomicrographic Tracings of the Nall-Fold Capillary Bed in a Patient With Confirmed Hemorrhagic Fever $(100 \mathrm{X})$ 


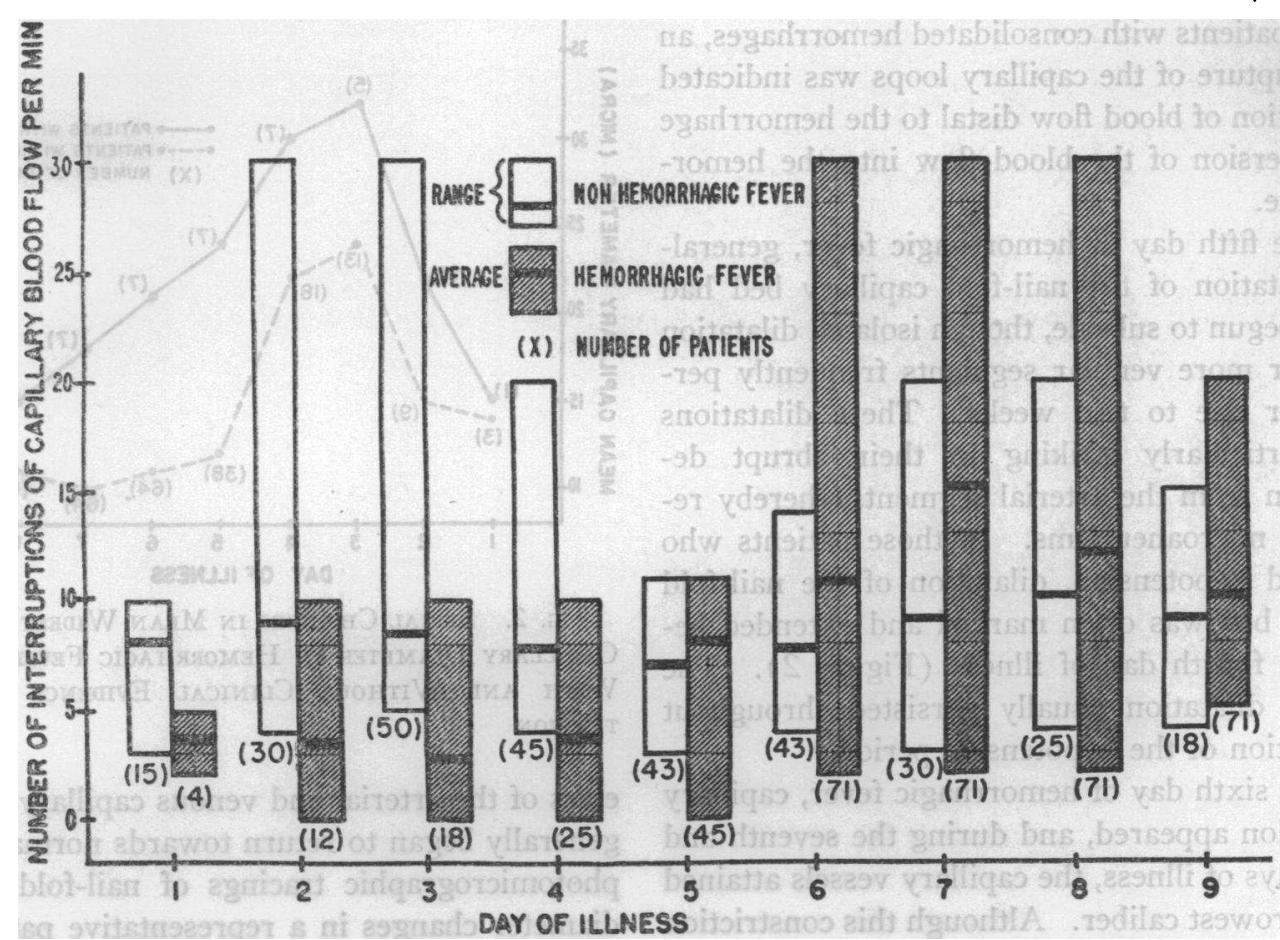

Fig. 4. Serial Observations of NaII-Fold Capillary Vasomotor Activity (and/or Plasma "Skmmming") in Patients With Hemorrhagic Fever and With the Other INFectious ILLNESSES Listed in TABLE I.

"gaps" in capillary blood flow were seen. If systemic arterial hypotension developed, the "gaps" in blood flow usually subsided entirely. By the fifth day of illness, the frequency of "gaps" in capillary blood flow increased, becoming most intense in those patients who developed systemic arterial hypertension, and then began to decrease towards normal by the ninth or tenth day of illness (Figure 4).

3) Blood flow. In most patients with hemorrhagic fever there were no marked alterations in the rate of capillary blood flow. However, during systemic arterial hypotension, a diminution in rate or actual stagnation of flow developed despite maintenance of warm extremities. During the hypertensive phase, rate of capillary blood flow increased above normal in approximately 30 per cent of the cases.

4) Sludging. Distribution of erythrocytes in the blood flowing through the capillary bed usually remained homogenous. In five patients with hemorrhagic fever, during the shock phase, 1 to 2 plus clumping of red blood cells was seen.

\section{B) Relation of nail-fold capillary changes to the} clinical course of hemorrhagic fever

1) The degree of capillary dilatation varied markedly from one patient to another, sometimes with no apparent relation to the severity of illness. Considered as a group, hovever, patients with a mild clinical course exhibited minimal or no capillary alterations, whereas patients with severe illness and shock developed more marked capillary dilatation (Table II).

2) Approximately 30 per cent of patients during the hypotensive phase of hemorrhagic fever failed to exhibit any increase in mean widest capillary diameter.

3) Decreased vasomotor activity (and/or plasma "skimming") was a more sensitive indicator of the presence of capillary involvement during the hypotensive phase of hemorrhagic fever than was an increase in mean widest capillary diameter. Although marked diminution or cessation of vasomotor activity invariably accompanied an increase in mean widest capillary diameter, one third of patients without detectable capillary dila- 
TABLE $\mathbf{I}$

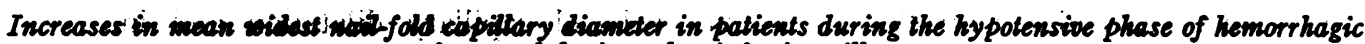
feoer and during ather infectious illnesses

\begin{tabular}{|c|c|c|c|c|c|c|c|c|c|c|c|c|}
\hline \multirow{2}{*}{$\frac{\text { Severity of clinical couree }}{\text { Diagnoels }}$} & \multicolumn{4}{|c|}{ Mild } & \multicolumn{4}{|c|}{ Moderate } & \multicolumn{4}{|c|}{ Severe } \\
\hline & $\begin{array}{l}\because \text { inos } \\
\text { cases }\end{array}$ & $\begin{array}{l}\text { Avge } \\
\text { (inticra) } \\
\text { (micas }\end{array}$ & $\begin{array}{l}\text { Range of } \\
\text { merteane } \\
\text { (micra) }\end{array}$ & $\begin{array}{l}\text { \% cases } \\
\text { with no } \\
\text { increase }\end{array}$ & $\underset{\text { cases }}{\text { No. }}$ & $\begin{array}{c}\text { Avg. } \\
\text { increase } \\
\text { (micra) }\end{array}$ & $\begin{array}{l}\text { Range of } \\
\text { increase } \\
\text { (micra) }\end{array}$ & $\begin{array}{l}\% \text { cases } \\
\text { with no } \\
\text { increase }\end{array}$ & $\underset{\text { cases }}{\text { No. }}$ & $\begin{array}{c}\text { Avg. } \\
\text { increase } \\
\text { (micra) }\end{array}$ & $\begin{array}{l}\text { Range of } \\
\text { increase } \\
\text { (micra) }\end{array}$ & $\begin{array}{l}\text { \% cases } \\
\text { with no } \\
\text { increase }\end{array}$ \\
\hline \multirow{5}{*}{$\begin{array}{l}\text { Hemorrhagic fever } \\
\text { Scrub typhus } \\
\text { Rocky Mt. spotted } \\
\text { fever } \\
\text { Infectious mono- } \\
\text { nucleosis } \\
\text { Generalized urticaria } \\
\text { following penicillin } \\
\text { given for upper re- } \\
\text { spiratory illness }\end{array}$} & 10 & 6 & \multirow[t]{5}{*}{$0-20$} & \multirow[t]{5}{*}{50} & 8 & 10 & \multirow[t]{5}{*}{$0-20$} & \multirow[t]{5}{*}{37} & \multirow[t]{5}{*}{7} & \multirow[t]{5}{*}{16} & \multirow[t]{5}{*}{$0-25$} & \multirow[t]{5}{*}{14} \\
\hline & 2 & 0 & & & 2 & 20 & & & & & & \\
\hline & 2 & 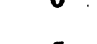 & & & 1 & 10 & & & & & & \\
\hline & 1 & 5 & & & & & & & & & & \\
\hline & 1 & 10 & & & & & & & & & & \\
\hline
\end{tabular}

tation exhibited a significant reduction in vasomotor activity. Stated another way, during the hypotensive phase of hemorrhagic fever, 70 per cent of the patients exhibited an increase in mean widest capillary diameter, whereas 80 per cent of the patients showed a reduction in vasomotor activity.

4) Subsidence of capillary dilatation or development of capillary constriction occurred at the time the blood urea nitrogen was rising rapidly and albuminuria was maximal (Figure 5).

5) Changes in venous hematocrit seemed to bear a distinct relation to changes in nail-fold capillary dilatation; thus, evidence of greatest plasma leakage as indicated by a rising hematocrit occurred during the phase of maximum capillary dilatation, and greatest resorption of plasma as indicated by a falling hematocrit occurred during maximum

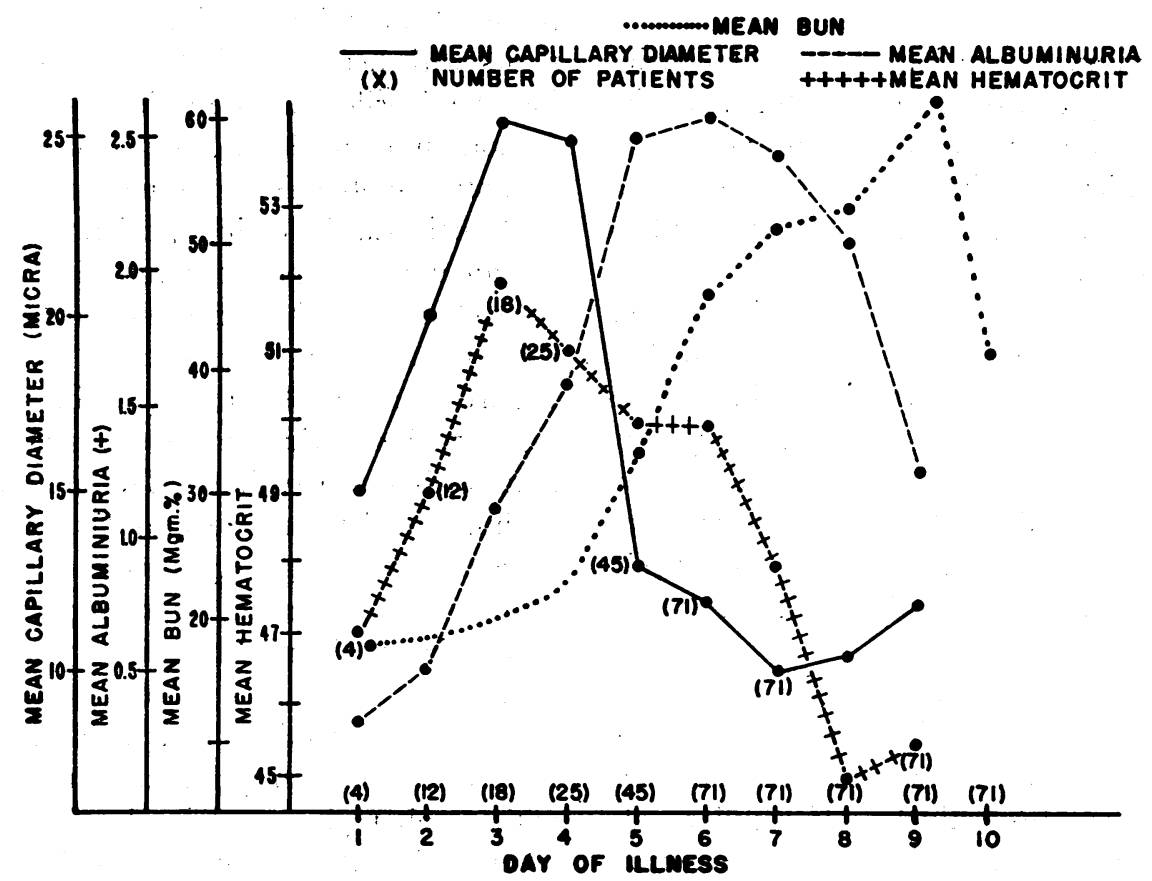

Fig. 5. Seril Determinations or Mean Widest NaIl-Fold Capillary Diameter, Mran Albumtuuria, Mean Blood Urea Nitrogen and Mran Venous Hzmatocrit in Patients With Hemorrhagic Fever 
capillary constriction (Figure 5). Since intake of fluids was usually carefully controlled to match total output (10), these hematocrit changes probably reflect alterations in general capillary permeability.

6) Gross conjunctival injection and facialthoracic flush persisted several days to one or two weeks after subsidence of the nail-fold capillary dilatation and remained unaltered despite the development of nail-fold capillary constriction. The conjunctival injection and facial-thoracic flush were also consistently observed in those patients exhibiting no evidence of nail-fold capillary dilatation or decreased vasomotor activity.

\section{C) Effect of various forms of therapy on nail-fold capillary alterations in hemorrhagic fever}

1) Tetracyclines in oral dosages of up to $4 \mathrm{Gms}$. daily did not significantly alter the nail-fold capillary changes.

2) The intravenous infusion of concentrated human albumin into five patients during the shock phase raised systemic arterial blood pressure and accelerated capillary blood flow but did not alter the other characteristics of the dilated nail-fold capillary bed.

3) The intravenous infusion of $l$-norepinephrine in quantities of up to 1.0 gamma per $\mathrm{Kg}$. per minute into 10 patients with hypotension and 8 nonhypotensive patients during the first afebrile day failed to significantly alter mean widest capillary diameter or vasomotor activity (and/or plasma "skimming"), although mean systemic arterial blood pressure was elevated by 10 to $24 \mathrm{~mm}$. $\mathrm{Hg}$. This is in sharp contrast to the effect in patients without infectious disease in whom capillary constriction and vasomotor activity is thereby increased markedly (23). Rate of capillary blood flow usually increased slightly with $l$-norepinephrine infusions, concomitant with the rise in arterial blood pressure.

4) Sixteen patients received oral cortisone for five successive days, beginning on the second or third day of illness with daily dosages of 300,200 , 200 , and $100 \mathrm{mg}$., respectively. If capillary abnormalities already existed when cortisone therapy was initiated, as seen in eight patients, there was no significant restorative effect. Six of the eight patients who received cortisone prior to the ap- pearance of vascular alterations subsequently developed capillary dilatation and impaired vasomotor activity (and/or plasma "skimming").

5) During the phase of maximum capillary dilatation, three patients were given single intravenous doses of $0.6 \mathrm{mg}$. per $\mathrm{Kg}$. of Benadryl over a five minute period. Rate of capillary blood flow and vasomotor activity (and/or plasma 'skimming") increased slightly. The mean widest capillary diameter decreased 5 micra in two patients. These changes were transient, persisting 10 to 30 minutes. In the one patient in whom this initial dose was followed by an intravenous infusion of Benadryl at a rate of $0.3 \mathrm{mg}$. per $\mathrm{Kg}$. per hour, the initial alteration in the capillary bed was not maintained.

\section{Febrile illnesses other than hemorrhagic fever}

Those febrile illnesses other than hemorrhagic fever that were studied for evidences of capillary alterations are indicated in Table I. Most of these patients, despite pyrexia of up to $105^{\circ} \mathrm{F}$., exhibited no consistent detectable nail-fold capillary changes. An increase in mean widest capillary diameter and decreased vasomotion (and/or plasma "skimming"), similar to that seen in patients with hemorrhagic fever, was observed during the febrile stages in single patients with infectious mononucleosis, scrub typhus, and Rocky Mountain spotted fever. One patient with generalized urticaria following penicillin administration for an upper respiratory infection also exhibited these changes. The increases in mean widest capillary diameter in these patients are indicated in Figure 1 and Table II. In addition, several pericapillary hemorrhages were observed in the patient with scrub typhus. The abnormal capillary findings persisted for several days following defervescence. Since only one patient with each of these illnesses was observed, no attempt is made to correlate the capillary alterations with the clinical course. It should be noted, however, that the patient with infectious mononucleosis developed a diffuse macular eruption and that the patients with scrub typhus and Rocky Mountain spotted fever both exhibited clinical and laboratory evidences of diffuse and severe injury to the capillary vascular system. Such capillary injury was indicated by a marked rise in venous hematocrit, pe- 


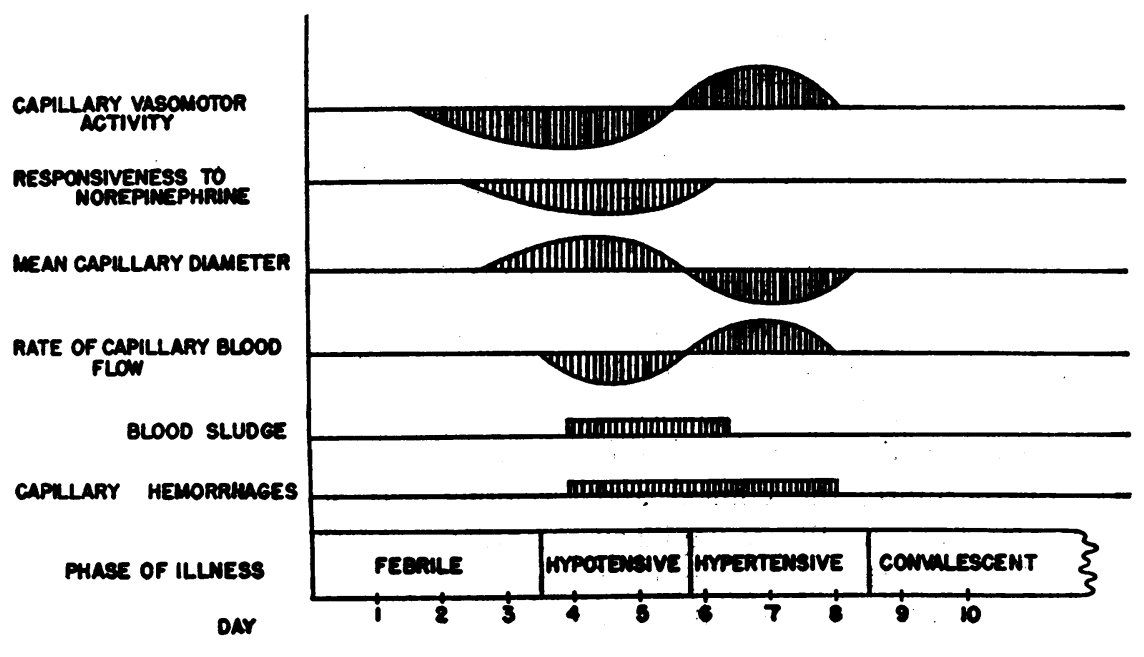

Fig. 6. Interpretive Summary of NaII-Fold Capillary Changes During HeMorRhagic FEVER

techial and hemorrhagic lesions on cutaneous and mucosal surfaces, strongly positive Rumpel-Leede tests, systemic arterial hypotension, and 2 to 3 plus albuminuria with microscopic hematuria, despite the institution of specific antibiotic therapy after approximately four days of illness. Moreover, the possibility that the severity and duration of the nail-fold capillary alterations may have been mitigated by the antibiotic therapy could not be adequately evaluated. In subsequent studies, two other patients with Rocky Mountain spotted fever were observed throughout the latter portion of the febrile phase and during convalescence. Despite pyrexia to $105^{\circ} \mathrm{F}$., neither patient exhibited any significant nail-fold capillary alterations. However, the cutaneous eruption in both cases was minimal in intensity and in neither of these patients was there any objective clinical or laboratory signs of generalized capillary injury.

\section{DISCUSSION}

By direct microscopy, alterations of the nail-fold capillary bed in patients acutely ill with various infectious diseases could be followed serially and correlated with the clinical course. Single observations were of limited value since the nail-fold capillary bed exhibited considerable individual variations. However, when considered collectively, the changes assumed certain patterns. For patients with hemorrhagic fever, the capillary alterations are presented as an interpretive summary (Figure 6). Arranged in order of frequency, decreased vasomotor activity (and/or plasma "skimming"), refractoriness to $l$-norepinephrine, dilatation, and hemorrhagic diathesis were the outstanding features observed in the nail-fold capillary bed during the febrile and hypotensive phases of illness. The decrease in vasomotor activity (and/or plasma "skimming"), the capillary dilatation, and the hemorrhagic diathesis, when compared with the control group of patients with other infectious illnesses, were significant (" $t$ " $>3.5$ for each alteration). The nail-fold capillary refractoriness to $l$-norepinephrine was also significant when contrasted with a group of patients with noninfectious diseases (23) studied previously (in the 0.16 to 0.20 gamma per $\mathrm{Kg}$. per minute range, " $t$ " equals 5.4). Insufficient data on the $l$-norepinephrine reactivity of the nail-fold capillary bed during other infectious illnesses precludes analysis as to the specificity of such capillary refractoriness for hemorrhagic fever. Slowing of capillary blood flow and sludging of erythrocytes during the febrile and hypotensive stages of hemorrhagic fever were not significant.

In contrast to the earlier phases of illness, increased vasomotor activity (and/or plasma "skimming") and vasoconstriction developed in the nailfold capillary bed during the hypertensive-oliguric and diuretic phases of hemorrhagic fever. These changes, when compared with those of the control group of patients with other infectious illnesses, were significant (" $t$ " $>2.6$ for each alteration). Similar alterations in the nail-fold capillary bed 
have been reported for hemorrhagic fever in Central Russia, probably the same disease as occurs in Korea (24).

It appears likely that the capillary changes observed during the course of hemorrhagic fever are not restricted to the nail-fold area, but rather reflect a general reaction pattern. Postmortem material from cases of hemorrhagic fever demonstrates that the initial dilatation of the nail-fold capillary vessels is not localized to the cutaneous area but that similar capillary dilatation occurs diffusely (12-14). Furthermore, the clinical and laboratory findings also indicate diffuse capillary dysfunction $(10,11)$. The close correlation of changes in venous hematocrit and nail-fold capillary diameter especially suggests that the nailfold capillary alterations may reflect generalized similar capillary changes. However, Russian investigators have recently demonstrated that the cutaneous capillaries of the chest and abdomen remain dilated during the phase of nail-fold capillary constriction and conclude that the capillary alterations during hemorrhagic fever are of a "segmental character" (24). Such regional dissociation of small vessel caliber during the later stages of hemorrhagic fever has been confirmed by the present study. Although the basis for this segmental capillary involvement is as yet undetermined, it seems unlikely that an infectious or humoral agent would constrict the capillary bed in one cutaneous area while simultaneously dilating comparable vessels in another cutaneous area. As will be indicated, it also seems improbable that regional variations in neurogenic factors produce the "segmental" capillary alterations. An alternative explanation which considers the regional variations in intensity of the initial capillary damage appears the most plausible. Although the capillary injury initiated during the febrile and hypotensive phases of hemorrhagic fever is generalized, the severity of this capillary injury is not uniform. Postmortem studies indicate that certain capillary areas such as the renal medulla, anterior pituitary, and right atrium are consistently involved more extensively than are most other areas $(12-14,25)$. Furthermore, 20 per cent of all patients with hemorrhagic fever exhibited no detectable nail-fold capillary alterations during the febrile and hypotensive phases despite concomitant clinical evidence of generalized capillary dysfunction. Even the intensity of the cutaneous capillary involvement appears to vary markedly in different regions since the small vessels of the face and thorax usually remained dilated for several days to one or two weeks following subsidence of the nail-fold capillary dilatation. Indeed, conjunctival and facial-thoracic capillary dilatation were consistently present in patients exhibiting no evidence of nailfold capillary dilatation. Since those capillary areas most severely injured may remain refractory to constrictor stimuli longer than other vascular regions less severely affected, the "segmental" capillary constriction during the latter stages of hemorrhagic fever may in part reflect regional differences in intensity of the initial small vessel injury.

The physiologic basis for the nail-fold capillary caliber alterations during hemorrhagic fever is uniknown. The initial capillary dilatation might be passive (i.e., increased "stretching") secondary to increased inflow of blood, impairment of outflow, or increased blood volume with "capillary storage" of blood such as described for polycythemia vera (19); or the capillary dilatation might be active due to loss of vascular tone. During the febrile and hypotensive phases of hemorrhagic fever, digital blood flow and antecubital venous pressures are often normal (26-28); blood volume is decreased (29). Such evidence suggests that the nail-fold capillary dilatation is related to loss of vascular tone, although the participation of venular constriction cannot be excluded. Similarly, the nail-fold capillary attenuation might be passive (i.e., diminished "stretching") secondary to decreased inflow of blood, facilitation of outflow, or decreased blood volume (antithesis of the "storage effect") ; or the capillary attenuation might be active due to increased vascular tone. During the hypertensive phase of hemorrhagic fever, digital blood flow may be increased above normal (26, $27)$; blood volume and antecubital venous pressures are generally normal $(28,29)$. Such evidence suggests that the nail-fold capillary attenuation results from an active increase in vascular tone.

The mechanism underlying the generalized capillary injury initiated early in the course of hemorrhagic fever is undetermined. It has been postulated that intense and generalized arteriolar dilatation per se might induce the diffuse capil- 
lary damage (25). It is unlikely, however, that the nail-fold capillary dilatation and reduction of vasomotion (and/or plasma "skimming") was secondary to cutaneous arteriolar dibtation since cutaneous arteriolar dilatation, as seen after cervicothoracic sympathectomy, results in a decrease in nail-fold capillary diameter (18). Moreover, during the infusion of $l$-norepinephrine for several days in 10 patients during the hypotensive phase of hemorrhagic fever, the fingers became cool and the nail-fold capillary circulation decreased. Despite these signs of digital arteriolar constriction, the nail-fold capillary loops remained dilated. Further evidence against the role of arteriolar dilatation is provided by observations that arteriolar dilatation fails to alter capillary diameter or vasomotor activity of the normal mammalian capillary bed $(18,30,31)$. Indeed, even the existence of generalized arteriolar dilatation in the usual patient with hemorrhagic fever seems improbable, since a widening of arterial pulse pressure, a decrease in peripheral resistance, and "capillary pulsations" were generally absent $(9-11,32)$. It appears more likely, therefore, that unidentified noxious factors act directly upon the capillary bed during the febrile and hypotensive phases of hemorrhagic fever. Attempts to define the presence of humoral capillary damaging factors in patients with hemorrhagic fever will be presented in a subsequent paper.

The etiology of the hypotensive phase of hemorrhagic fever has received considerable study. A generalized loss of arteriolar tone, or inability of the arterioles to respond to constrictor stimuli, suggested as a possible causative mechanism (25), did not appear responsible as peripheral resistance was found elevated in most patients with hypotension (32). Generalized capillary dilatation, however, may induce arterial hypotension by reducing effective circulating blood volume $(33,34)$. A further reduction of effective circulating blood volume, induced by plasma leakage through damaged capillary walls, would be favored by a decrease in capillary vasomotor activity (35). Thus, two mechanisms for the systemic arterial hypotension during hemorrhagic fever-capillary dilatation and loss of vasomotor activity-are reflected in the changes seen in the nail-fold capillary bed.

Compared to the development of nail-fold capillary dilatation over a 48 to 96 hour period, con- striction of the capillary bed usually developed within 24 to 48 hours, paralleling the rapid appearance of hypertensive systemic arterial blood pressure levels. Nail-fold capillary vasomotor activity (and/or plasma "skimming") increased simultaneously. Such increase of vasomotor activity, if generalized, would favor resorption of interstitial fluid (35), and might account in part for the rapid fall in hematocrit during this phase. Since present evidence suggests that neurogenic factors play no significant role in the production of nail-fold capillary constriction or enhanced vasomotion $(18,36)$, and since the fingers of patients during the phase of nail-fold capillary constriction remained warm and capillary blood flow remained rapid, it seems unlikely that neurogenic factors were primarily responsible for the nail-fold capillary constriction. The role of humoral factors in the production of the nail-fold capillary constriction, however, is unknown. Although the hypertensive state and the nail-fold capillary constriction usually developed at the time albuminuria was maximum and the blood urea nitrogen was increasing rapidly, the participation of renal vasoconstrictor substances remains speculative and unproven.

An agent that mitigates the diffuse capillary damage in hemorrhagic fever has not been found. The tetracyclines, Benadrylo, cortisone, concentrated serum albumin, and $l$-norepinephrine did not significantly prevent or reverse the nail-fold capillary dilatation or loss of vasomotor activity (and/ or plasma "skimming"). These agents, moreover, failed to materially alter the clinical course of the illness $(11,37,38)$.

The majority of patients acutely ill with infectious diseases other than hemorrhagic fever, despite pyrexia to $105^{\circ} \mathrm{F}$., failed to exhibit any significant nail-fold capillary alterations. However, in single patients with scrub typhus and infectious mononucleosis, the serial nail-fold capillary findings during the febrile stages were indistinguishable from those during the febrile and hypotensive phases of hemorrhagic fever. Comparable capillary changes also developed in one patient with Rocky Mountain spotted fever who exhibited concomitant clinical evidence of severe and generalized small vessel injury. The nail-fold capillary alterations during the febrile and hypotensive phases of hemorrhagic fever are therefore not specific, but 
appear to represent a basic vascular response pattern to a variety of infectious agents. Whereas the mechanism for these capillary changes during hemorrhagic fever remain unknown, such alterations during scrub typhus and Rocky Mountain spotted fever may be explained, in part at least, by the direct rickettsial invasion of the vessel wall (7). As with hemorrhagic fever, it seems likely that the capillary alterations in the patients with scrub typhus and Rocky Mountain spotted fever are not peculiar to the nail-fold area but reflect the diffuse small vessel injury which characterizes most rickettsial infections (7). Although $Q$ fever is classified as a rickettsial infection, clinically and pathologically this disease is very different from the other rickettsial infections. In man, no important lesions are found outside the lungs; the characteristic diffuse involvement of small blood vessels, including those of the skin such as occurs with the other rickettsial agents, are conspicuously absent (7). It appears significant, therefore, that no consistent nail-fold capillary alterations were noted in the nine patients clinically ill with $Q$ fever. However, since these patients all received specific antibiotic therapy after the first day of fever (15), the possibility of capillary alterations occurring later in the untreated disease cannot be excluded. The significance of the nail-fold capillary changes in the patient with infectious mononucleosis is unknown. Although petechial hemorrhages may appear on the hard palate (39), the presence of the cutaneous eruption and the absence of any pathological description or clinical evidence of visceral capillary injury in this disease (40-42) suggest that the capillary changes in this patient may have been limited to the cutaneous area.

\section{SUMMARY}

Alterations of the nail-fold capillary vessels of 71 patients with hemorrhagic fever and 51 patients with a variety of other infectious illnesses have been followed serially by direct microscopy:

\section{A) Hemorrhagic fever}

1. Arranged in order of frequency, decreased vasomotor activity (and/or plasma "skimming"), refractoriness to $l$-norepinephrine, increase in mean widest diameter, and hemorrhagic diathesis were the outstanding features observed during the fe- brile and hypotensive phases. Slowing of capillary blood flow and sludging of erythrocytes were less conspicuous.

2. The intensity of the capillary dilatation and loss of vasomotor activity (and/or plasma "skimming") paralleled the severity of the clinical course, although considerable variation occurred in individual cases. Twenty per cent of the patients failed to exhibit any such capillary alterations in the nail-fold area despite concomitant clinical and laboratory evidences of diffuse capillary injury.

3. The dilatation and loss of vasomotor activity (and/or plasma "skimming") in the nail-fold capillary bed were not prevented nor significantly reversed by administration of tetracyclines, Benadrylo, cortisone, serum albumin, or $l$-norepinephrine.

4. The mean widest capillary diameter returned toward normal, in the average patient, by the fifth day of illness. Constriction of the nail-fold capillary bed and heightened vasomotor activity (and/ or plasma "skimming") subsequently appeared during the hypertensive-oliguric and diuretic phases of illness.

5. The alterations of the capillary vessels are not peculiar to the nail-fold area; rather they appear to reflect the presence of injurious factors acting diffusely and directly, although not uniformly, upon the capillary vascular system.

\section{B) Other infectious illnesses}

1. Most patients with infectious illnesses other than hemorrhagic fever, despite pyrexia to $105^{\circ}$ F., exhibited no nail-fold capillary alterations.

2. The nail-fold capillary changes in two patients with clinical evidence of diffuse and severe capillary injury during scrub typhus and Rocky Mountain. spotted fever were indistinguishable from those seen during the febrile and hypotensive phases of hemorrhagic fever. Comparable alterations were also noted in one patient with infectious mononucleosis associated with cutaneous manifestations.

3. Nine patients with early $Q$ fever, in contrast to the patients with the other rickettsial diseases studied, exhibited no significant alterations of the nail-fold capillary vessels. These differences may be related to differences in the clinical 
and pathological findings in the various rickettsial infections.

4. Of the nail-fold capillary changes observed during the febrile and hypotensive phases of hemorrhagic fever and during the course of the other infectious illnesses, none were specific for any given disease; indeed such changes appear to represent a general response pattern to a number of infectious agents that act primarily upon the capillary vascular system. Nail-fold capillary constriction and heightened vasomotor activity (and/or plasma "skimming"), however, as occurred during the hypertensive phase of hemorrhagic fever, appeared to be specific for this infectious illness.

\section{REFERENCES}

1. Snyder, J. C., The typhus fevers in Viral and Rickettsial Infections of Man, T. M. Rivers, Ed, 2nd ed. Philadelphia, J. B. Lippincott Co., 1952, p. 578.

2. Cox, H. R., The spotted-fever group in Viral and Rickettsial Infections of Man, T. M. Rivers, Ed., 2nd ed. Philadelphia, J. B. Lippincott Co., 1952, p. 611.

3. Smadel, J. E., Scrub typhus in Viral and Rickettsial Infections of Man, T. M. Rivers, Ed., 2nd ed. Philadelphia, J. B. Lippincott Co., 1952, p. 638.

4. Schoenbach, E. B., The meningococci in Bacterial and Mycotic Infections of Man, R. J. Dubos, Ed., 2nd ed. Philadelphia, J. B. Lippincott Co., 1952, p. 547.

5. Weinman, D., The bartonella group in Bacterial and Mycotic Infections of Man, R. J. Dubos, Ed., 2nd ed. Philadelphia, J. B. Lippincott Co., 1952, p. 608.

6. Banks, H. S., Meningococcosis. A protean disease. Lancet, 1948, 2, 635.

7. Rickettsial Diseases of Man, F. R. Moulton, Ed. Washington, American Association for the Advancement of Science, 1948.

8. Earle, D. P., Analysis of sequential physiologic derangements in epidemic hemorrhagic fever. Am. J. Med., 1954, 16, 690.

9. Sheedy, J. A., Froeb, H. F., Batson, H. A., Conley, C. C., Murphy, J. P., Hunter, R. B., Cugell, D. W., Giles, R. B., Bershadsky, S. C., Vester, J. W., and Yoe, R. H., The clinical course of epidemic hemorrhagic fever. Am. J. Med., 1954, 16, 619.

10. Barbero, G. J., Katz, S., Kraus, H., and Leedham, C. L., Clinical and laboratory study of thirty-one patients with hemorrhagic fever. Arch. Int. Med., 1953, 91, 177.

11. Leedham, C. L., Epidemic hemorrhagic fever: a summarization. Ann. Int. Med., 1953, 38, 106.

12. Hullinghorst, R. L., and Steer, A., Pathology of epidemic hemorrhagic fever. Ann. Int. Med., 1953, 38, 77.
13. Kessler, W. H., Gross anatomic features found in 27 autopsies of epidemic hemorrhagic fever. Ann. Int. Med., 1953, 38, 73.

14. Lukes, R. J., The pathology of thirty-nine fatal cases of epidemic hemorrhagic fever. Am. J. Med., 1954, $16,639$.

15. Tigertt, W. D., and Benenson, A. S., Studies on $Q$ fever in man. Trans. A. Am. Physicians, 1956, 69, 98.

16. Crawford, J. H., Studies on human capillaries. II. Observations on the capillary circulation in normal subjects. J. Clin. Invest., 1925-1926, 2, 351.

17. Greisman, S. E., Unpublished observations.

18. Brown, G. E., Observations on the surface capillaries in man following cervicothoracic sympathetic ganglionectomy. J. Clin. Invest., 1930, 9, 115.

19. Brown, G. E., and Sheard, C., Measurements on the skin capillaries in cases of polycythemia vera and the rôle of these capillaries in the production of erythrosis. J. Clin. Invest., 1925-1926, 2, 423.

20. Crawford, J. H., Studies on human capillaries. III. Observations in cases of auricular fibrillation. $\mathrm{J}$. Clin. Invest., 1925-1926, 2, 365.

21. Greisman, S. E., The reactivity of the capillary bed of the nailfold to circulating epinephrine and norepinephrine in patients with normal blood pressure and with essential hypertension. J. Clin. Invest., 1952, 31, 782.

22. Knisely, M. H., Bloch, E. H., Eliot, T. S., and Warner, L., Sludged blood. Science, 1947, 106, 431.

23. Greisman, S. E., The reaction of the capillary bed of the nailfold to the continuous intravenous infusion of levo-nor-epinephrine in patients with normal blood pressure and with essential hypertension. J. Clin. Invest., 1954, 33, 975.

24. Chumakov, M. P., Reznikov, A. I., Dzagurov, S. G., Leshchinskaia, E. V., Glazunov, S. L., Dubniakova, A. M., and Povalishina, T. P., Hemorrhagic fever with renal syndrome in the Upper Volga basin. Voproay Virusologii, 1956, 4, 26.

25. Wood, W. B., Jr., Clinical aspects of epidemic hemorrhagic fever. Report to Surgeon General of visit to Hemorrhagic Fever Center in Korea, 18 September-14 October, 1952.

26. McClure, W. W., Plethysmographic studies in epidemic hemorrhagic fever. Preliminary observations. Am. J. Med., 1954, 16, 664.

27. Lyons, R. H., Syner, J., and Moe, G., Hemodynamics of epidemic hemorrhagic fever. Tr. Am. Clin. \& Climatol. A., 1954, 66, 48.

28. Cugell, D. W., Cardiac output in epidemic hemorrhagic fever. Am. J. Med., 1954, 16, 668.

29. Giles, R. B., and Langdon, E. A., Blood volume in epidemic hemorrhagic fever. Am. J. Med., 1954, $16,654$.

30. Webb, R. L., and Nicoll, P. A., Persistence of active vasomotion after denervation. Federation Proc., 1952, 11, 169. 
31. Wiedeman, M. P., Reactivity of arterioles following denervation of subcutaneous areas of the bat wing. Am. J. Physiol., 1954, 177, 308.

32. Entwhisle, G., and Hale, E., Hemodynamic alterations in hemorrhagic fever. Circulation, 1957, 15, 414.

33. Greisman, S. E., The regulation of effective circulating blood volume. Med. Bull. of the U. S. Army, Far East, 1954, 2, 32.

34. Moon, V. H., Circulatory failure of capillary origin. J. A. M. A., 1940, 114, 1312.

35. Chambers, R., and Zweifach, B. W., Functional activity of the blood capillary bed, with special reference to visceral tissue in The Annals of the New York Academy of Science. New York, New York Academy of Science, 1946, vol. 46, p. 683.

36. Greisman, S. E., The reaction of the nail-fold capillary bed during the cold pressor response, Unpublished observations.
37. Stockard, J. L., Hale, E. H., and Bullard, H. V., Diphenhydramine therapy of epidemic hemorrhagic fever: in the early febrile phase. U. S. Armed Forces Med. J., 1956, 7, 1405.

38. Sayer, W. J., Entwhisle, G., Uyeno, B., and Bignall, R. C., Cortisone therapy of early epidemic hemorrhagic fever: a preliminary report. Ann. Int. Med., 1955, 42, 839.

39. Holzel, A., An early clinical sign of infectious mononucleosis. Lancet, 1954, 267, 1054.

40. Boyd, W., A Textbook of Pathology, 6th ed. Philadelphia, Lea \& Febiger, 1953.

41. Ziegler, E. E., Infectious mononucleosis. Report of a fatal case with autopsy. Arch. Path., 1944, 37, 196.

42. Allen, F. H., Jr., and Kellner, A., Infectious mononucleosis. An autopsy report. Am. J. Path., 1947, 23, 463. 